\title{
Protective Effects of Antimuscarinics on the Bladder Remodeling After Bladder Outlet Obstruction
}

\author{
Qiang Liu Deyi Luo Tongxin Yang Banghua Liao Hong Li Kunjie Wang
}

Department of Urology, Institute of Urology (Laboratory of Reconstructive Urology), West China Hospital, Sichuan University, Chengdu, Sichuan, P.R.China

\section{Key Words}

Bladder outlet obstruction • Antimuscarinics • Muscarinic receptor

\begin{abstract}
Background/Aims: Overactive bladder associated with bladder outlet obstruction (BOO) is a highly prevalent condition, which is usually treated with antimuscarinics. However, the potential effects of antimuscarinics on the structure and function of bladder have not been investigated thus far. Methods: Sprague-Dawley(R) rats accepted bladder neck obstruction surgery or sham surgery, and then received treatment of three different antimuscarinics (Solifenacin, Darifenacin, and Tolterodine) or vehicle. After 3, 6 and 12 weeks, the bladder function and structure were measured. The effect of antimuscarinics on cellular alteration in vitro was observed under mechanical stimulation. Bladder morphology were examined by immunohistochemistry, and the bladder function were investigated by cystometry and strip contractility test. The expression of muscarinic receptors and inflammatory cytokines were measured by PCR and Western blotting. Results: Here we demonstrate, both in vitro and in vivo, that antimuscarinics are protective regulators for the bladder structure and function. Antimuscarinics decrease the weight of bladders with BOO. Antimuscarinics improve the voiding parameter and enhance the contraction of bladder smooth muscle. The results also show that antimuscarinics inhibit the proliferation of bladder smooth muscle cells both in vivo and in vitro, it can reduce the collagen deposition and inflammatory cytokines in bladders with BOO. During this process, the expression of M2 and M3 receptors was altered by antimuscarinics. Conclusion: Antimuscarinics could reverse the structural and functional changes of $\mathrm{BOO}$ bladder wall at cellular and tissue level, and the alteration of M2 and M3 receptors may be involved in this biological process.
\end{abstract}

(C) 2017 The Author(s)

Published by S. Karger AG, Basel

\section{Introduction}

Bladder overactivity, which is characterized by symptoms of urinary urgency, frequency and nocturia, with or without urinary incontinence [1], occurs in $45-50 \%$ of patients with D. Luo and Q. Liu contributed equally to this work.

Kunjie Wang

KARGER
Department of Urology, Institute of Urology (Laboratory of Reconstructive Urology), West China Hospital, Sichuan University, Chengdu, Sichuan, (P.R. China) Tel. +86-158-2825 0943, Fax 86-28-8542 2451, E-Mail wangkj@scu.edu.cn 
bladder outlet obstruction (BOO)[2]. Notably, the prevalence and incidence of BOO and bladder overactivity are increasing in the context of the aging of global population. By 2030, $20 \%$ of the US population will be 65 years or older, including over 20 million men [3].

Antimuscarinic drugs are widely applied in this category of patients and had been demonstrated to be effective and safe [4]. The mechanism by which antimuscarinics work is that such kind of drugs can interrupt urothelial sensory and local contractions of the bladder wall mediated by muscarinic receptors [5]. However, the potential effects of antimuscarinics on structural and functional changes of bladders caused by BOO have not been explored so far. Nevertheless, recent findings implicate that muscarinic receptors are able to enhance the cell proliferation, modulate contractile protein expression and participate in the release of inflammatory cytokines [6-9]. But, up to date, the role of antimuscarinics and muscarinic receptors in bladder function and structure is still unclear.

Accordingly, this study aimed to investigate the effect of antimuscarinics on bladder remodeling using rat BOO model. As mechanical tension within the bladder after BOO increased, therefore, we also study the effect of antimuscarinics on the cell biological characteristics under mechanical stimuli in vitro.

\section{Materials and Methods}

\section{Animals and BOO model}

Eight-week-old female Sprague-Dawley rats, weighing 220 to 250 g were used for the study. All animal experiments were carried out in adherence with the National Institutes of Health Guidelines on the Use of Laboratory Animals and approved by the West China Hospital Committee on Animal Care.

The rats were anesthetized by intraperitoneal injection with $0.9 \%$ chloral hydrate $(3 \mathrm{ml} / \mathrm{Kg})$.Then a suprapubic midline incision was made to expose the bladder and proximal urethra. An epidural catheter (outer diameter: $1 \mathrm{~mm}$ ) was inserted in the urethra, and then was tied with 4-0 silk around the proximal urethra to create a partial B00. The catheter was then removed, and the abdominal incision was closed. The sham group received the same procedure except for the urethra ligation.

The rats were divided into 3-, 6-, 12-week group according to the time of obstruction. The rats were randomly sub-divided into 5 groups: the sham group, BOO group, BOO with Solifenacin $(0.45 \mathrm{mg} / \mathrm{kg}), \mathrm{BOO}$ with Darifenacin $(0.68 \mathrm{mg} / \mathrm{kg})$, and BOO with Tolterodine $(0.36 \mathrm{mg} / \mathrm{kg})$ group. All drugs were dissolved in distilled water and administrated by gavage. The dosage was determined based on the equivalent dose of clinical use (Solifenacin 5mg/kg, Darifenacin $7.5 \mathrm{mg} / \mathrm{kg}$, and Tolterodine $4 \mathrm{mg} / \mathrm{kg}$ ). The sham group and BOO group received the same amount of distilled water. Treatment was initiated at the first day after surgery, and continued once daily for the entire experiment.

\section{Bladder weight measurement and histologic analysis.}

The rats were sacrificed and the bladder harvested for bladder weight measurement and histology. Five bladder specimens from each group were fixed in $4 \%$ paraformaldehyde for $24 \mathrm{~h}$. All the bladder specimens were taken from equivalent part which was orientated in the same manner for sectioning. For the collagen analysis, 5 randomly areas from the slide were captured to analyze the collagen area. After that, the samples were paraffin-embedded, and cut into 4-um sections. The slides were stained with Masson's trichrome to examine the smooth muscle and collagen area. To study the collagen area, images from each slide were captured using a digital camera (Olympus, Japan) in 5 randomly chosen areas at 200 magnifications. The areas of collagen (blue-stained) and muscle tissue (red-stained) were quantified using Image-Pro Plus (Media Cybernetics, MD). Then the mean percent collagen area (collagen/collagen+muscle) was calculated.

\section{Cytometry experiments}

Rats were anesthetized by intraperitoneal injection of urethane $(1 \mathrm{~g} / \mathrm{kg})$. The bladder was exposed though a midline suprapubic incision. Then a $25 \mathrm{G}$ needle connecting to a polyethylene catheter was inserted into the bladder dome, and then connected to a pressure transducer and a microinjection pump via a three-way connector. Cytometry was performed by infusing warm saline at a rate of $0.04 \mathrm{~mL} / \mathrm{min}$. After stabilization of micturition cycle, the cytometry parameters were monitored, including micturition pressure 


\section{Cellular Physiology Cell Physiol Biochem 2017;44:907-919 \begin{tabular}{l|l} 
and Biochemistry Published online: November 24, 2017 & $\begin{array}{l}\text { (c) 2017 The Author(s). Published by S. Karger AG, Basel } \\
\text { www.karger.com/cpb }\end{array}$ \\
\hline DOI.1159/00048358
\end{tabular}}

Liu et al.: Antimuscarinics Prevent Bladder Remodeling

(maximum bladder pressure during micturition, MP), baseline pressure (lowest bladder pressure during filling, BP), threshold pressure (bladder pressure immediately before micturition, TP) and micturition interval (MI). Five rats from each group were tested.

Functional studies of bladder muscle

Six rats from each group were used for organ-bath studies. The bladder was cut longitudinally from the bladder neck to the dome, and then the mucosa was removed. The smooth muscle strips measuring $2 \times 10 \mathrm{~mm}$ were prepared in the Krebs solution(mMNaCl 117.7, $\mathrm{KCl} 4.69, \mathrm{CaCl} 22.16, \mathrm{MgSO} 41.20$, NaHCO 324.39 , KH2PO 41.2 and glucose $9.99 ; 95 \% 02 / 5 \% \mathrm{CO} 2,37^{\circ} \mathrm{C}, \mathrm{pH} 7.4$ ). Each strip was connected to a force displacement transducer, and washed with Krebs every 15 minutes. The muscle strip contractile response was induced by high $\mathrm{K}^{+}$solution $(80 \mathrm{mM})$ and cumulative addition of carbachol. The tension was normalised to tissue weight.

HBSMCs culture and cell proliferation assay under stretch stimulation

Human bladder smooth muscle cells (HBSMCs: ScienCell, Carlsbad, CA, USA; cat. no. 4310) were expanded in culture using Dulbecco's modified Eagle's medium (DMEM; low glucose) supplemented with $10 \%$ fetal bovine serum (both Hyclone Laboratories, Inc., Logan, UT, USA), penicillin $(100 \mathrm{U} / \mathrm{ml})$ and streptomycin $(100 \mu \mathrm{g} / \mathrm{ml})$. These cells were maintained at $37^{\circ} \mathrm{C}$, in a humidified atmosphere of $5 \% \mathrm{CO} 2 / 95 \%$ air in a cell incubator. For mechanical stimuli, HBSMCs were seeded onto a silicone membrane and subjected to cyclic stretch using the Bose BioDynamic (Bose Corporation, Eden Prairie, MN, USA). The treated silicone membrane was subjected to $0.1 \mathrm{~Hz}$ stretch at $10 \%$ equibiaxial elongation for $6 \mathrm{~h}$. A silicone membrane without stretch was used as control. To assess the possible underlying mechanisms, M receptor antagonists were applied: 4-DAMP (sc-200167) for the M3 receptor antagonist, AF-DX116 (sc-223772) for the M2 receptor antagonist and atropine for the non-selective antagonist (All from Santa Cruz Biotechnology, Inc., Santa Cruz, CA, USA). All the treated cells were subjected to stretch stimulation. HBSMCs from each group were harvested and suspended at a concentration of $4 \times 10^{5}$ cells $/ \mathrm{ml}$ in DMEM. The cell suspension was transferred into a 96-well plate, with $200 \mu \mathrm{l}$ in each. $10 \mu \mathrm{M}$ BrdU labeling reagent was added and the cells were reincubated for $3 \mathrm{~h}$. Then cells were fixed and exposed to anti-BrdU antibody (1:100). Proliferation was quantified by measuring the absorbance value at a wavelength of $450 \mathrm{~nm}$ using an uQuant ELISA microplate reader (BioTek Instruments, Inc., Winooski, VT, USA).

\section{Immunohistochemistry.}

For immunohistochemistry study, five rats from each group were examined. Cell proliferation marker was detected using the following primary antibody: PCNA [Santa Cruz Biotechnology, Santa Cruz, CA, USA, 1:200 dilution]. All the bladder specimens were taken from equivalent part which was orientated in the same manner for sectioning and five randomly chosen representative areas from the slide were captured and analyzed using Image-Pro Plus software.

Real-time PCR analysis of gene expression

Five bladder specimens from each group were examined. The mRNA levels of CCL2, CCL5, IL6, M2, and M3 gene were examined via quantitative real-time PCR. Total RNA was extracted using TRIzol reagent and cDNA was synthesized using SuperScript II (Takara Bio, Inc., Shiga, Japan). Real-time PCR was completed using the SYBR Premix Ex Taq (Takara Bio, Inc.) and the Bio-Rad iQ5 machine (Hercules, CA, USA). The
Table 1. The sequences of primers for quantitative real-time PCR

\begin{tabular}{lcr}
\hline Gene & & PCR primers \\
\hline GAPDH & Forward & 5'-GTGGTGCCAAAAGGGTCA-3' \\
& Reverse & 5'-ATTTCTCGTGGTTCACACCCA-3' \\
M2 & Forward & 5'-CCACTCCAGAGAGATGACAACT-3' \\
& Reverse & 5'-GGCTACAACGTTCTGCTTT-3' \\
M3 & Forward & 5'-GGACTGTGGATGTGGAGAG-3' \\
& Reverse & 5'-CGAGGAGTTGGTGTCAGA-3' \\
CCL2 & Forward & 5'-GATGCAGTTAATGCCCCACT-3' \\
& Reverse & 5'-TTCCTTATTGGGGTCAGCAC-3' \\
CCL5 & Forward & 5'-TGTCCCACTTGAAGGAGTAT-3' \\
& Reverse & 5'-ATCCCCAGCTGGTTAGGACT-3' \\
IL6 & Forward & 5'-ACCATGTTGCCGTTCCTAAG-3' \\
& Reverse & 5'-CAGCATGTTCTTCCCCTCAT-3' \\
\hline
\end{tabular}


conditions of PCR were programmed as $94^{\circ} \mathrm{C}$ for 3 min and 40 cycles of $94^{\circ} \mathrm{C}$ for $5 \mathrm{sec}, 54^{\circ} \mathrm{C}$ for $30 \mathrm{sec}$ and $72^{\circ} \mathrm{C}$ for $20 \mathrm{sec}$. The product quality was monitored using post-PCR melt curve analysis. Glyceraldehyde3-phosphate dehydrogenase (GAPDH) is the housekeeper gene which was measured for normalization of samples. The sequences of primers for quantitative real-time PCR are listed in Table 1.

\section{Western blotting}

The protein levels of M2 and M3 were examined using western blot and five bladder specimens from each group were examined. The frozen tissue was pulverized and homogenized at $4^{\circ} \mathrm{C}$. Homogenates were centrifuged at $13000 \mathrm{rpm}$ for 20 minutes at $4^{\circ} \mathrm{C}$, the pellet discarded, and the supernatant was used immediately. After electrophoresis, the proteins were transferred to polyvinylidene fluoride membranes for 2 hours. Nonspecific binding sites were blocked by incubation for 1 hour 1 Tween 20/Tris-buffered saline containing $5 \%$ skim milk and $0.1 \%$ Tween 20 . Membranes were then incubated with primary antibodies overnight. The primary antibodies anti-mAChR2 and anti-mAChR3 purchased from Santa Cruz Biotechnology (Santa Cruz, CA, USA, 1:100). Membranes were incubated with rabbit immunoglobulin G secondary antibody horseradish peroxidase (1:2000; Abcam Plc) for 1 hour after washing. Membranes were also probed with an anti-glyceraldehyde-3-phosphate dehydrogenase monoclonal antibody. Then, protein signals were detected by exposure on BioMax MR-1 film (Kodak, Rochester, NY, USA). Expression levels were calculated by measuring the density of the bands. The protein bands were quantified as a ratio to GAPDH using Image-Pro Plus.

\section{Statistical analysis}

Results are presented as means and standard deviations (SD). The data were analyzed statistically using ANOVA, followed by a Scheffe post hoc test for multiple comparisons. Differences with $\mathrm{p}<0.05$ were defined significant.

\section{Results}

Antimuscarinics decreased the weight of bladder with $\mathrm{BOO}$

To explore the role of antimuscarinics in the structure of bladder, the change of bladder weight was firstly observed. The average bladder weight of the BOO groups was significantly heavier than that of the sham group( $303 \pm 47$ vs $89.0 \pm 2.4)$ at 3 weeks, $650 \pm 66$ vs $91.9 \pm 3.8$ at 6 weeks, and $874 \pm 121$ vs $93.2 \pm 1.6$ at 12 weeks, respectively; $(\mathrm{P}<0.001)$ (Fig.1). All the B00 groups treated with different antimuscarinics showed decreased bladder weight compared with the BOO group. At 3 weeks, the average bladder weight in the Solifenacin, Darifenacin, and Tolterodine group were $283 \pm 44,291 \pm 49$, and $287 \pm 31$, respectively. However, the difference of bladder weight was not significant $(\mathrm{P}>0.05)$ (Fig. 1). At 6 weeks, the average bladder weight in $\mathrm{BOO}$ groups treated with Solifenacin, Darifenacin, and Tolterodine were $563 \pm 72,568 \pm 91$, and $573 \pm 83(\mathrm{P}<0.05$ vs $\mathrm{BO0}$ group). At 12 weeks, it was $777 \pm 101,773 \pm 51$, and $797 \pm 71$, respectively $(\mathrm{P}<0.05$ vs $\mathrm{BOO}$ group). No significant difference was found among the Solifenacin, Darifenacin, and Tolterodine groups at 3, 6 and 12 weeks ( $\mathrm{P}>0.05$, Fig. 1).

Antimuscarinics changed the bladder voiding parameters

Besides, the function of bladder was studied. The result of cystometry was obtained at 3 and 6 weeks, normal voiding

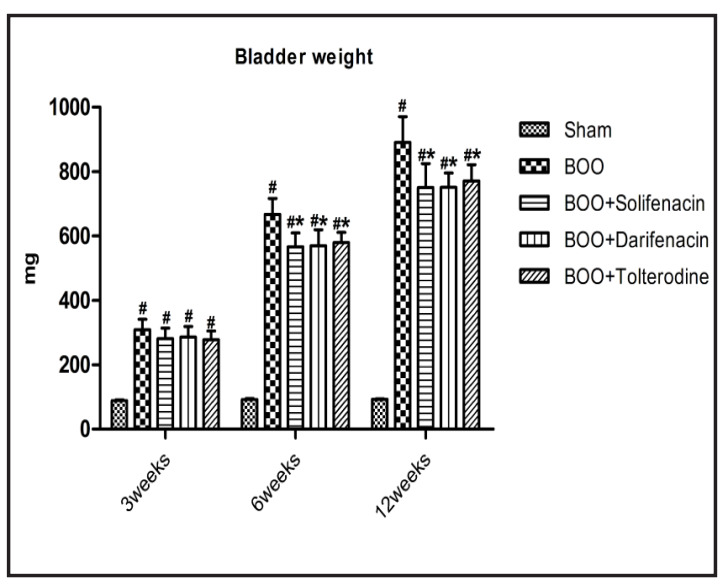

Fig. 1. Antimuscarinics decrease the weight of bladder with B00. Bladder is weighted after BOO or $\mathrm{BOO}+$ antimuscarinics at 3, 6, 12 weeks. Data are given as mean \pm s.d, $n=10-12$ for each group. Error bars represent s.d. 


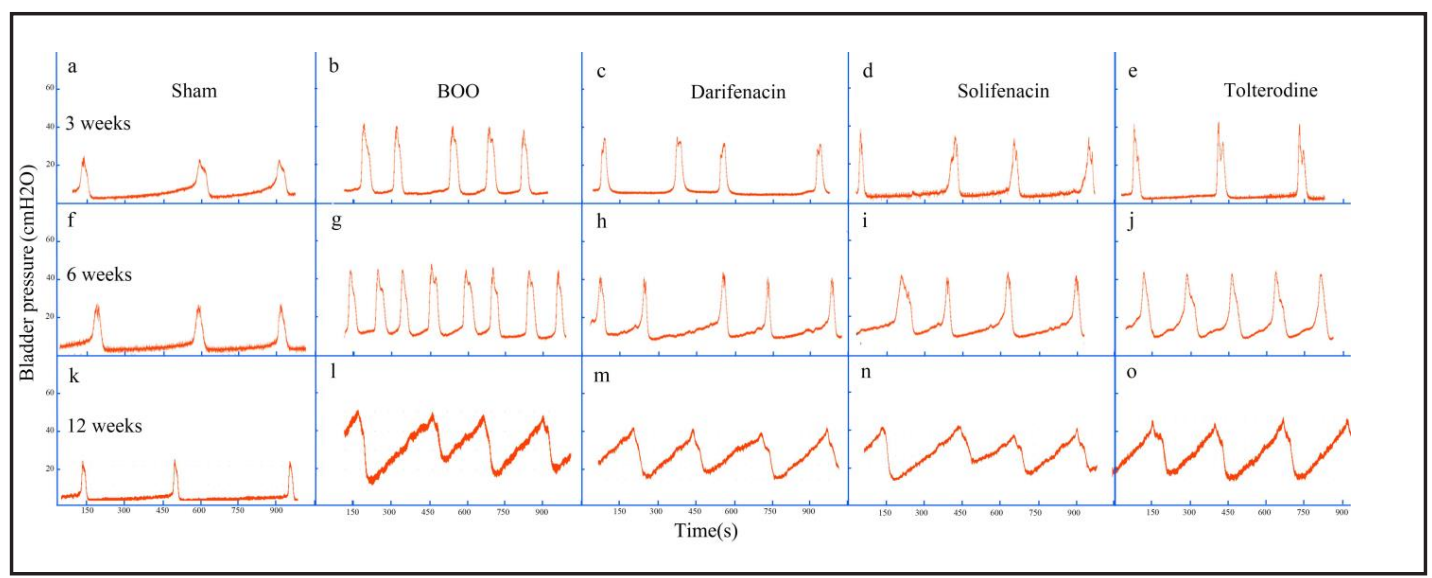

Fig. 2. Effect of antimuscarinics on cystometry parameters of rats with BO0. The bladder pressure (in centimeters of water) during continuous cystometry is recorded by cystometrogram. (a-e) The sample traces for sham, BOO, BOO with Solifenacin, BOO with Darifenacin, and BOO with Tolterodine group at 3 weeks. (f-j) The sample traces for sham, BOO, BOO with Solifenacin, BOO with Darifenacin, and BOO with Tolterodine group at 6 weeks. (k-o) The sample traces for sham, B00, BOO with Solifenacin, B00 with Darifenacin, and BOO with Tolterodine group at 12 weeks.

pattern was unable to be observed at 12 weeks because overflow was found by cystometry in all the obstructed rats. The micturition interval (second) in the BOO group was shorter than that in the sham group (174 \pm 52.54 vs $440 \pm 98$ at 3 weeks, $\mathrm{P}<0.01 ; \quad 122.83 \pm 33.10$ vs $433 \pm 125.4$ at 6 weeks, $\mathrm{P}<0.01$; Fig. 2, Table 2). In all the BOO treated with antimuscarinics groups, the micturition interval was longer compared with the BOO group at 3 and 6 weeks $(\mathrm{P}<0.05$; Fig. 2, Table 2). At
Table 2. The changes of bladder voiding parameters in sham group, BOO group and BOO+ antimuscarinics group at 3 and 6 weeks. B00: Bladder outlet obstruction; BP: Basline pressure (lowest bladder pressure during filling); TP: Threshold pressure (bladder pressure immediately before micturition); MP: micturition pressure (maximum bladder pressure during micturition); Maxium MI(s): Micturition interval (s); $a$ : $\mathrm{p}<0.05$ versus sham; $\mathrm{b}$ : $\mathrm{p}<0.05$ versus $\mathrm{BO0}, \mathrm{n}=6$

\begin{tabular}{lcccc}
\hline Group & BP $(\mathrm{cmH} 20)$ & TP $(\mathrm{cmH} 20)$ & MP $(\mathrm{cmH} 20)$ & MI $(\mathrm{s})$ \\
\hline 3 weeks & & & & \\
Sham & $2.03 \pm 0.83$ & $7.29 \pm 1.8$ & $24.98 \pm 1.8$ & $440 \pm 98$ \\
BOO & $6.05 \pm 2.6^{\mathrm{a}}$ & $12.6 \pm 3.03^{\mathrm{a}}$ & $39.7 \pm 2.99^{\mathrm{a}}$ & $174 \pm 52.54^{\mathrm{a}}$ \\
BOO+ Darifenacin & $4.09 \pm 2.01^{\mathrm{a}}$ & $10.86 \pm 1.60^{\mathrm{a}}$ & $38.67 \pm 4.80^{\mathrm{a}}$ & $301.30 \pm 85.16^{\mathrm{ab}}$ \\
BOO+ Solifenacin & $4.50 \pm 0.57^{\mathrm{ab}}$ & $9.49 \pm 0.97^{\mathrm{a}}$ & $35.95 \pm 4.88^{\mathrm{a}}$ & $297.80 \pm 104.81^{\mathrm{ab}}$ \\
BOO+ Tolterodine & $4.38 \pm 0.47^{\mathrm{ab}}$ & $9.84 \pm 1.71^{\mathrm{a}}$ & $35.89 \pm 2.61^{\mathrm{a}}$ & $282.90 \pm 59.91^{\mathrm{ab}}$ \\
6 Weeks & & & & \\
Sham & $2.11 \pm 0.87$ & $6.7 \pm 2.26$ & $25.39 \pm 2.67$ & $433 \pm 125.4$ \\
BOO & $13.14 \pm 4.22^{\mathrm{a}}$ & $19.48 \pm 6.42^{\mathrm{a}}$ & $47.36 \pm 3.50^{\mathrm{a}}$ & $122.83 \pm 33.10^{\mathrm{a}}$ \\
BOO+ Darifenacin & $10.05 \pm 3.04^{\mathrm{a}}$ & $18.20 \pm 3.09^{\mathrm{a}}$ & $44.95 \pm 8.65^{\mathrm{a}}$ & $220.00 \pm 39.00^{\mathrm{ab}}$ \\
BOO+ Solifenacin & $10.23 \pm 2.58^{\mathrm{a}}$ & $17.18 \pm 6.13^{\mathrm{a}}$ & $44.35 \pm 9.28^{\mathrm{a}}$ & $201.4 \pm 48.31^{\mathrm{ab}}$ \\
BOO+ Tolterodine & $11.16 \pm 1.77^{\mathrm{a}}$ & $18.92 \pm 2.30^{\mathrm{a}}$ & $46.17 \pm 4.344^{\mathrm{a}}$ & $200.00 \pm 51.54 \mathrm{ab}$ \\
\hline & & & & \\
\hline
\end{tabular}
3 and 6 weeks, micturition pressure, baseline pressure and threshold pressure were higher in the BOO group compared with sham group $(\mathrm{P}<0.05)$. As showed in Table 2, these parameters in $\mathrm{BOO}$ groups treated with antimuscarinics were lower than the parameters in the BOO group at 3 and 6 weeks. No significant difference was found in the bladder pressure and micturition interval among the BOO treated with antimuscarinics groups at 3 and 6 weeks ( $>0.05)$.

\section{Antimuscarinics enhance the contraction of the smooth muscle of bladder}

To verify further the role of antimuscarinics in the bladder function, the contraction of the smooth muscle of bladder was investigated. At 3 weeks, the $\mathrm{KCl}$ produced contraction in BO0 group was $101.2 \%$ of that in the sham group ( $P>0.05$ ). At 6 and 12 weeks, obstruction resulted in a significant decrease of contraction in the responses to $\mathrm{KCl}(87.6 \%, 73.1 \%$ of that in the sham group, $\mathrm{P}<0.001)$. At 3 weeks, in the BOO group treated with Solifenacin, 


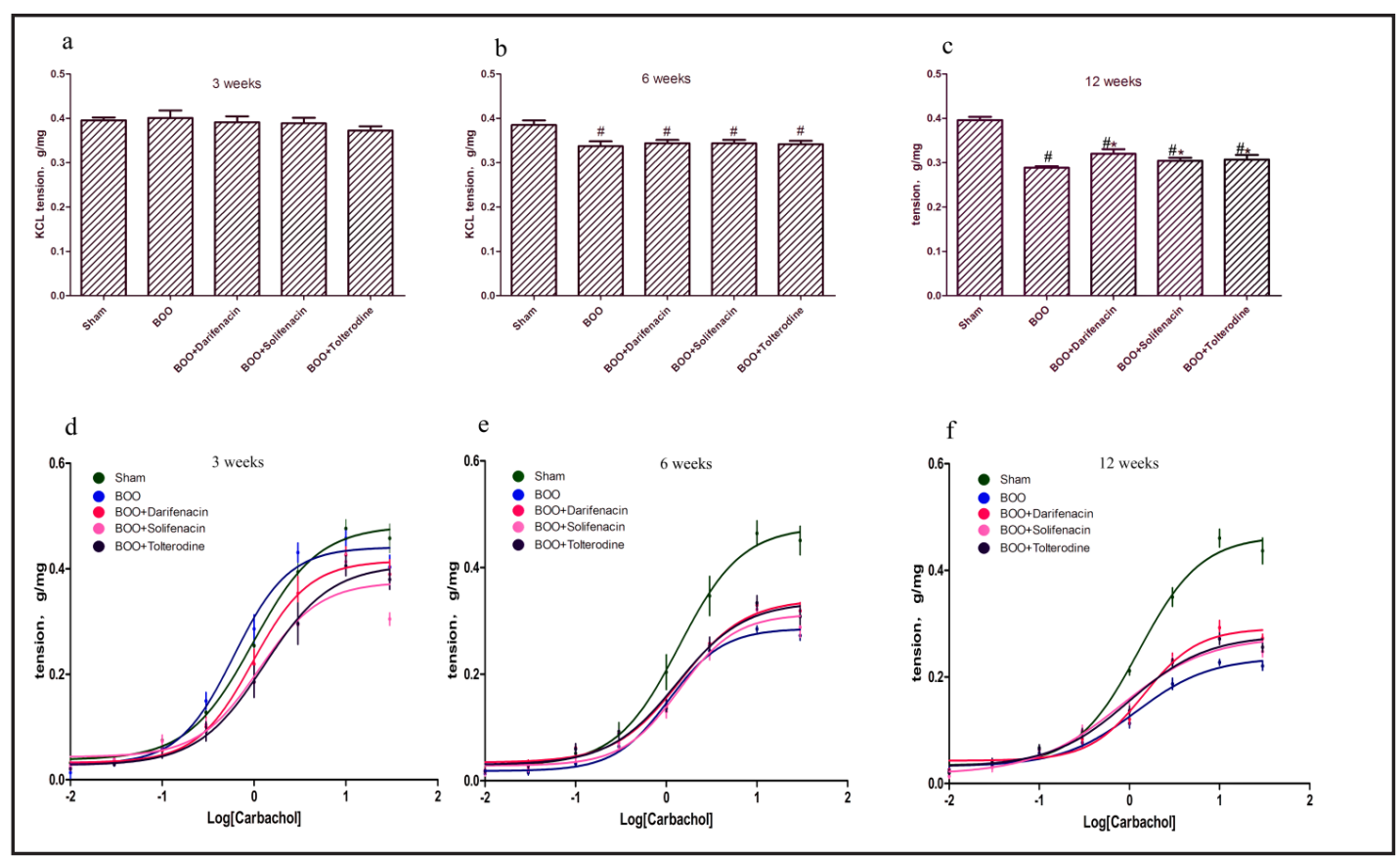

Fig. 3. Functional investigations of isolated bladder smooth muscle. The histograms showed the contractile response to $80 \mathrm{mM}$ potassium chloride. $(\mathrm{a}, \mathrm{b}, \mathrm{c})$ the contractile response to potassium chloride at 3,6 , and 12 weeks. Data are given as mean \pm s.d, \# $\mathrm{p}<0.001$ versus sham, ${ }^{*} \mathrm{p}<0.05$ versus $\mathrm{B00}, \mathrm{n}=6$. (d,e,f) the concentration-response curve to carbachol (10-7-10-4M) at 3, 6, and 12 weeks. Error bars indicate s.d, $\mathrm{n}=6$.

Darifenacin, and Tolterodine, the $\mathrm{KCl}$ induced contraction was $98.7 \%, 98.2 \%$, and $94.2 \%$ of that in the sham group $(\mathrm{P}>0.05)$, but there was no significant difference compared with the $\mathrm{BOO}$ group $(\mathrm{P}>0.05)$. At 6 weeks and 12 weeks, the $\mathrm{KCl}$ produced stronger contraction in BOO groups treated with Solifenacin, Darifenacin, and Tolterodine compared with BOO group $(1.8 \%, 1.7 \%$, and $1.4 \%$ increase at 6 weeks, $P>0.05 ; 5.3 \%, 10.8 \%$ and $6.3 \%$ increase at 12 weeks, $\mathrm{P}<0.05$ ). No significant difference was found among the Solifenacin, Darifenacin, and Tolterodine groups at 3, 6 and 12 weeks.

At 3, 6, and 12 weeks, the response to carbachol in the BO0 group was $95.1 \%, 61.3 \%$, and $49.4 \%$ of that in the sham group ( $\mathrm{P}<0.01)$. At 3 weeks, it was $91.8 \%, 86.9 \%$, and $86.2 \%$ in B00 groups treated with Solifenacin, Darifenacin, and Tolterodine group. There was no significant difference when compared with the BO0 group ( $P>0.05)$. At 6 and 12 weeks, the response was significantly stronger in the $\mathrm{BOO}$ with Solifenacin, Darifenacin, and Tolterodine group, showing an increase of $10.3 \%, 10.6 \%$, and $10.7 \%$ at 6 weeks and $19.7 \%, 28.9 \%$ and $19.2 \%$ at 12 weeks compared with B00 group ( $\mathrm{P}<0.05$, Fig. 3 ). No significant difference of response to carbachol was found among the Solifenacin, Darifenacin, and Tolterodine groups at 3 , and 6 weeks. At 12 weeks, the group treated with Darifenacin showed more obvious response to $\mathrm{KCl}$ and carbachol, however, compared with the Solifenacin and Tolterodine group, there was no significant difference $(\mathrm{P}>0.05)$.

\section{Antimuscarinics inhibited the HBSMCs proliferation in vivo and in vitro}

The cell proliferation and collagen deposition may contribute to remodeling of bladder. Therefore, the cell proliferation was validated in vivo and in vitro. In vivo, the proliferation of smooth muscle cell of rat bladder was examined by PCNA. At 3 weeks, the proliferation index increased from $5 \%$ in sham group to $17.4 \%$ in BO0 group $(\mathrm{P}<0.01)$. The proliferative index in the BOO group treated with antimuscarinics was lower than that in the BOO group at 3 weeks (14\% in Tolterodine group, $13.4 \%$ in Solifenacin group, and $16.1 \%$ in Darifenacin group, respectively; $\mathrm{P}<0.05$ ). At 6 and 12 weeks, no significant difference was found in 


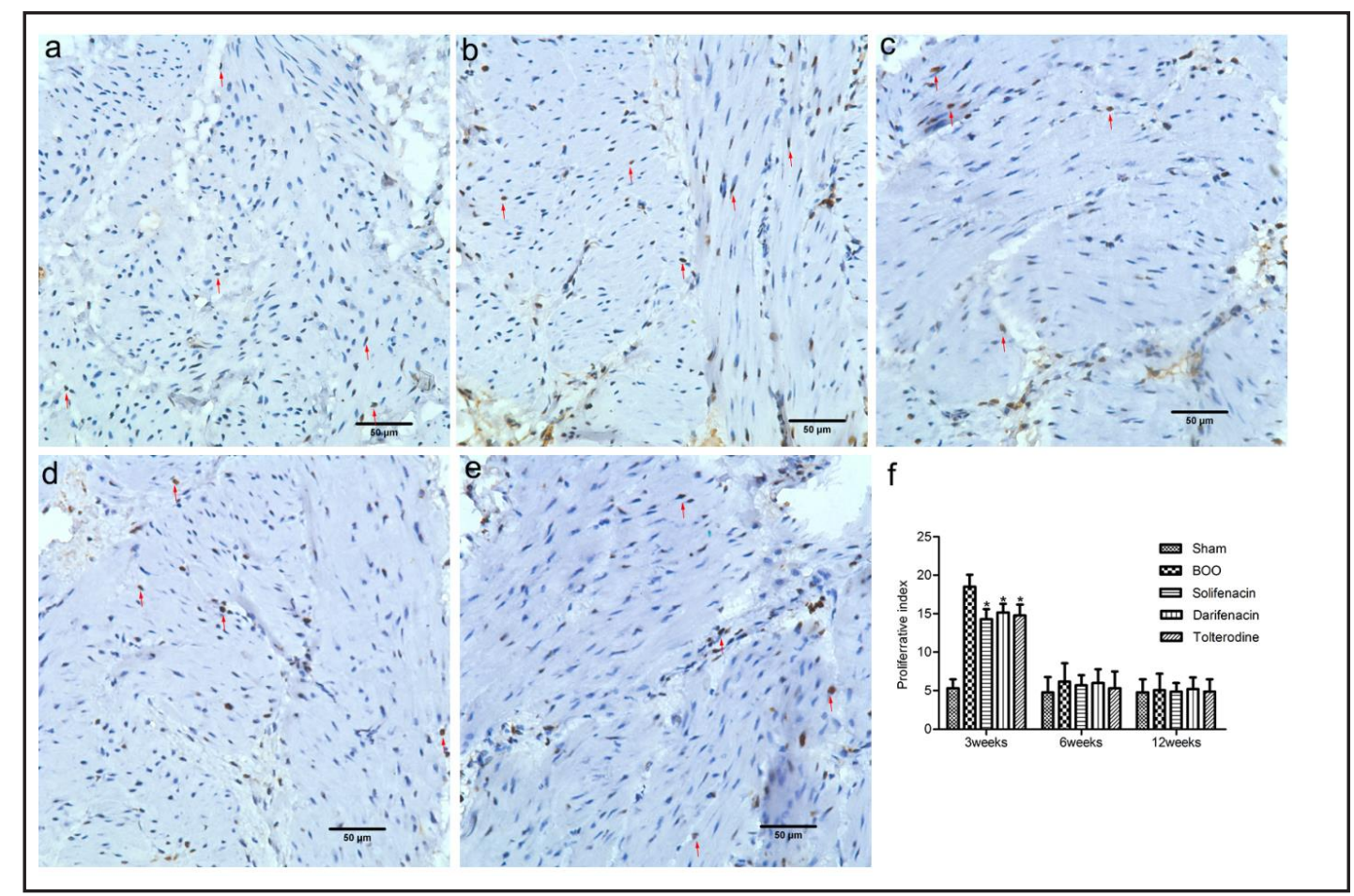

Fig. 4. The effect of antimuscarinics on cell proliferation. The pictures indicated the immunoreactivity of cells for PCNA (a cell proliferation marker, brown) in the sham (a), BOO (b), BOO with Solifenacin (c), BOO with Darifenacin (d), and BOO with tolterodine (e) group at 3 weeks. The red arrows show PCNA positive cells, Magnification 200. (f) The calculated proliferative index in each group. Values of bars graphs are given as mean \pm s.d, ${ }^{*} p<0.05$ versus BOO, $n=5$.

Fig. 5. Effect of muscarinic antagonists on BrdU incorporation into human bladder smooth muscle cells. The cells in the control group were cultured without stretch stimulation. The mechanical stimuli group was under stretch stimulation without treatment. The treated cells were subjected to the same stretch stimulation with different antimuscarinics. Data are given as mean $\pm \mathrm{s} . \mathrm{d},{ }^{*} \mathrm{p}<0.05$ versus control.

proliferative index in all groups ( $\mathrm{P}>0.05$, Fig. 4 ).

In vitro, the effect of antimuscarinics on HBSMCs proliferation under mechanical stimuli was

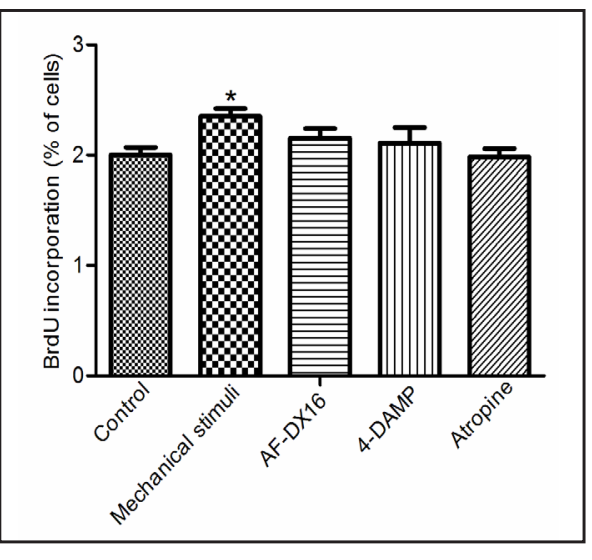
examined. Proliferation index of HBSMCs increased by $17.6 \%$ when exposed to mechanical stimuli. In the presence of antimuscarinics of AFDX16, 4-DAMP and atropine, proliferation was reduced by $8.4,10.2$, and $15.8 \%$, respectively ( $P>0.05$, Fig. 5).

Antimuscarinics reduced the collagen deposition in bladder with BOO after 12 weeks

Then, the change of collagen deposition in bladder was investigated. At 3 weeks, the BOO group displayed lower percent collagen area (\%) compared to the sham group $(16.97 \pm 1.4$ vs $21.85 \pm 1.2, \mathrm{P}<0.05)$. At 6 weeks, there was no significant difference between B00 group and sham group $(22.29 \pm 2.0$ vs $21.76 \pm 1.5, P>0.05)$. At 12 weeks, the BOO group displayed significantly higher percent collagen area compared with sham group $(32.06 \pm 3.0$ vs 
Fig. 6. Histologic analysis of bladder morphology in BOO. Masson trichrome staining was used to analyze collagen (stained blue) and smooth muscle (stained red). The pictures are the representative images in the sham $(a, f, k)$, BOO (b,g,l), BOO with Solifenacin $(c, h, m), B O 0$ with Darifenacin $(\mathrm{d}, \mathrm{i}, \mathrm{n})$, and B00 with tolterodine $(\mathrm{e}, \mathrm{j}, \mathrm{o})$ group at 3,6 ,and 12 weeks. Magnification 100.

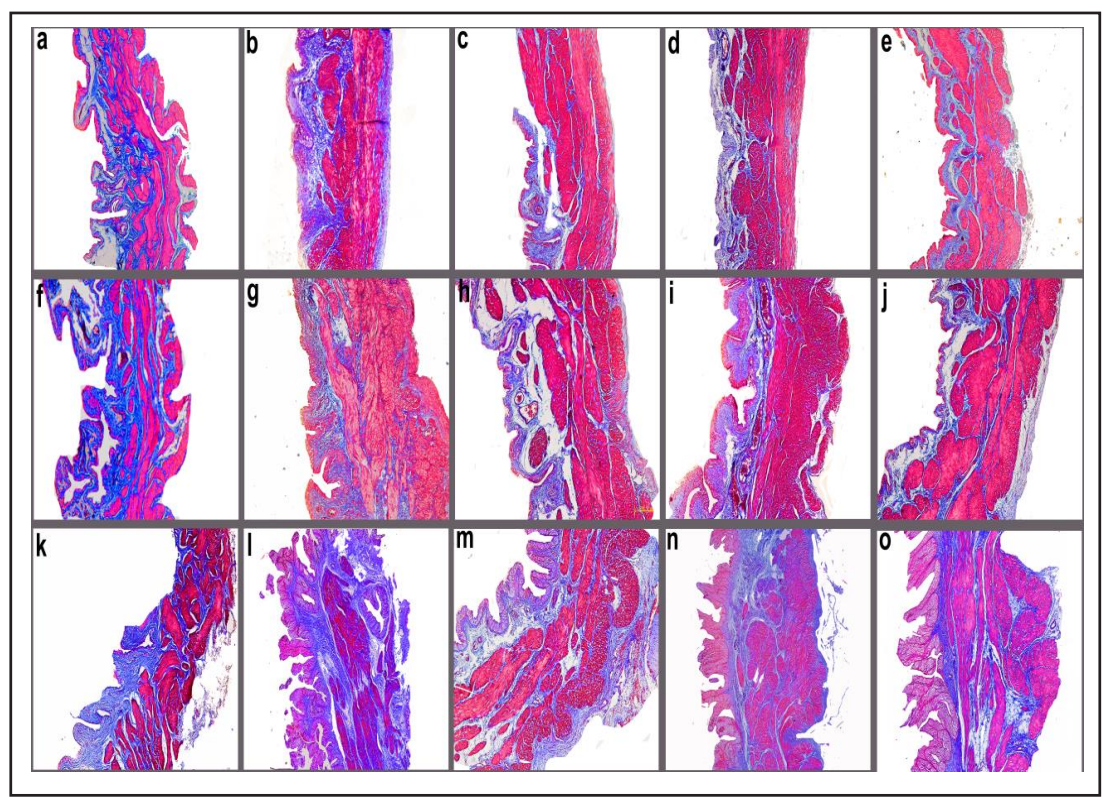

$22.14 \pm 1.3, \quad \mathrm{P}<0.05)$ ). At 3 and 6 weeks, the percent collagen area in BOO with Solifenacin, Darifenacin, and Tolterodine group had no significantly difference in comparison with BOO group $(16.85 \pm 1.0,17.0 \pm 1.7$, and $16.24 \pm 1.5$ at 3 weeks, $26.79 \pm 2.0$, $26.27 \pm 1.6$, and $25.77 \pm 1.2$ at 6 weeks; P>0.05 vs $\mathrm{BO0}$ group). At 12 weeks, however, a significant reduction of percent collagen area in BOO with Solifenacin, Darifenacin,

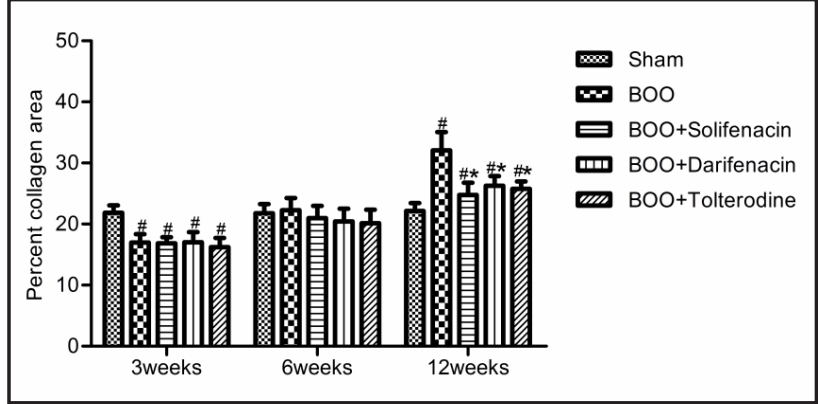

Fig. 7. Percent collagen area of bladder wall after BOO. Data are given as mean $\pm s . d,{ }^{*} p<0.05$ versus BOO, $\# p<0.05$ versus sham, $\mathrm{n}=5$. and Tolterodine group was observed $\quad(24.79 \pm 2.0,26.27 \pm 1.6$, and $25.77 \pm 1.2$, respectively; $\mathrm{P}<0.05$ vs $\mathrm{BO0}$ group). No significant difference was found among the Solifenacin, Darifenacin, and Tolterodine groups at 3, 6 and 12 weeks (Fig. 6, 7).

\section{Antimuscarinics lessened the inflammatory cytokines}

Also, the bladder remodeling along with the alteration of inflammatory cytokines, whether antimuscarinics can reduce the expression of inflammatory cytokines was testified. The CCL2 gene expression in BO0 group was 2.7, 6.4, and 4.8-fold higher compared with that in the sham group at 3,6 , and 12 weeks $(\mathrm{P}<0.001)$. The mRNA level of CCL2 in the B00 with Solifenacin, Darifenacin, and Tolterodine group was 1.0, 0.19, and 1.18-fold higher at 3 weeks, 4.7, 2.4, and 2.5-fold at 6 weeks, and 2.2, 1.2, and 2.2 fold at 12 weeks (Fig. 8). Darifenacin had lower CCL2 gene expression compared to Solifenacin and Tolterodine with significant difference at 3 weeks $(\mathrm{P}<0.05)$, and no significant difference was found at 6 and 12 weeks.

Similarly, the expression of CCL5 was 8.0,13.6, and 3.0-fold compared to that in the sham group at 3,6, and 12 weeks. At 3 and 6 weeks, all antimuscarinics can significantly suppressed the CCL5 expression ( $\mathrm{P}<0.05$ vs BO0 group). At 12 weeks, only Darifenacin, and Tolterodine can inhibit the CCL5 expression $(\mathrm{P}<0.05)$. At 3 and 6 weeks, no significant difference was found among the Solifenacin, Darifenacin, and Tolterodine groups $(\mathrm{P}>0.05)$, At 12 weeks, 


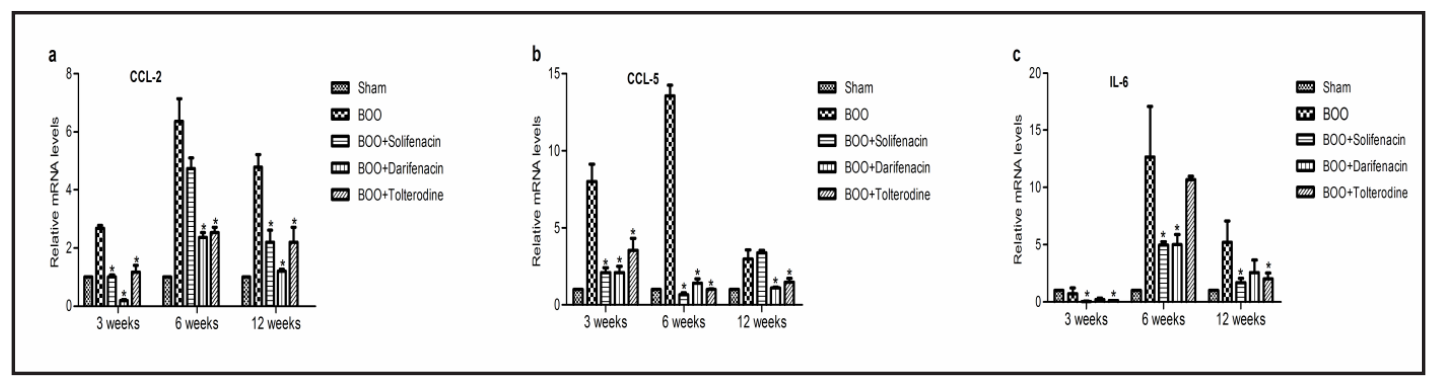

Fig. 8. The gene expression of inflammatory cytokines. Real-time reverse transcription-PCR (rRT-PCR) analyses was used to detect the expression of CCL2 (a), CCL5(b) and IL6(c).Data are given as mean \pm s.d,* $\mathrm{p}<0.05$ versus $\mathrm{BO0}, \mathrm{n}=5$.

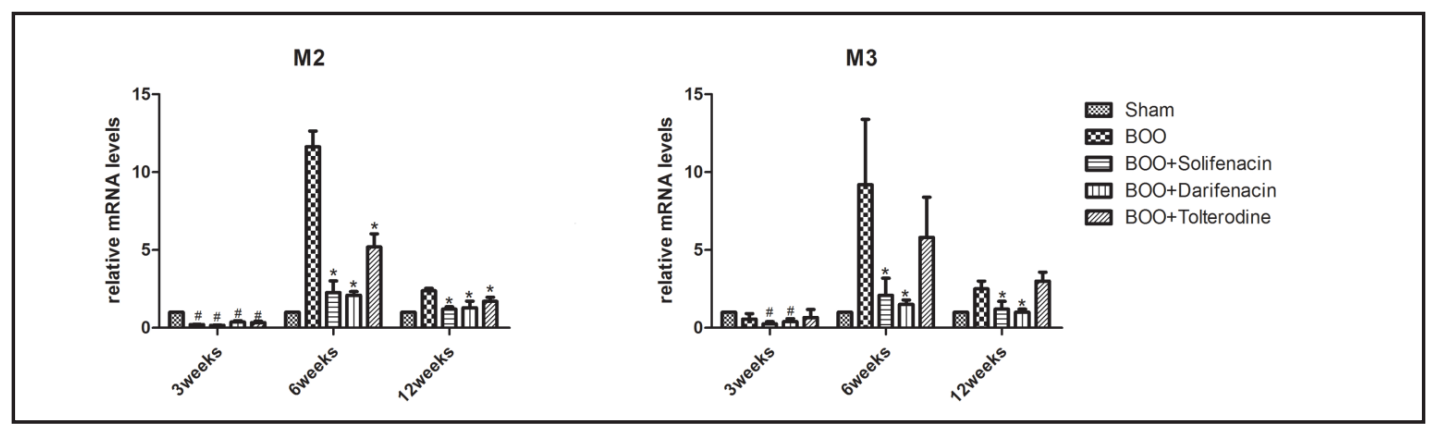

Fig. 9.The mRNA levels of M receptor. (a)The rRT-PCR analyses for M2 receptor. (b)The rRT-PCR analyses for $\mathrm{M} 3$ receptor. Data are given as mean $\pm \mathrm{s} . \mathrm{d},{ }^{*} \mathrm{p}<0.05$ versus $\mathrm{B} 00, \mathrm{~B} \mathrm{p}<0.05$ versus sham, $\mathrm{n}=5$.

the expression of CCL5 in Solifenacin group was higher than that in the Darifenacin, and Tolterodine group $(\mathrm{P}<0.05)$.

At 3 weeks, the mRNA level of IL6 was lower in the B00 group which was $70 \%$ of that in the sham group $(\mathrm{P}<0.05)$.At 6 and 12 weeks, it was upregulated in $\mathrm{B} 00$ group, which was 12 and 5.3 fold of that in the sham group. At 3 weeks, the groups with Solifenacin and Tolterodine treatment displayed significantly lower mRNA level compared to the BOO group $(\mathrm{P}<0.05)$. At 6 weeks, the groups treated with Solifenacin and Darifenacin showed lower IL6 expression compared with BOO group ( $\mathrm{P}<0.05$, Fig. 8). At 12 weeks, the groups with Solifenacin and Tolterodine treatment had same trend with that at 3 weeks. At 3 and 12 weeks, no significant difference was found among the Solifenacin, Darifenacin, and Tolterodine groups $(\mathrm{P}>0.05)$, At 6 weeks, the expression of IL6 in Tolterodine group was higher than that in the Darifenacin and Solifenacin group $(\mathrm{P}<0.05)$.

\section{Antimuscarinics altered the expression of M2 and M3 receptor}

At last, to explore the mechanism of antimuscarinics inhibited the bladder remodeling, the expression of $\mathrm{M} 2$ and $\mathrm{M} 3$ receptor was measured. The mRNA levels of $\mathrm{M} 2$ receptor in the BO0 group was $0.2,11.6$ and 2.37-fold of that in the sham group at 3,6 and 12 week $(\mathrm{P}<0.01)$. At 3 weeks, there was no difference between the BOO with and without antimuscarinics group for the $\mathrm{M} 2$ receptor expression $(\mathrm{P}>0.05)$. The groups with antimuscarinics treatment could significantly suppress M2 receptor expressions at 6 and 12 weeks $(\mathrm{P}<0.05)$. At 3 and 12 weeks, no significant difference was found among the Solifenacin, Darifenacin, and Tolterodine groups $(\mathrm{P}>0.05)$. At 6 weeks, the expression of M2 in Tolterodine group was higher than that in the Darifenacin and Solifenacin group $(\mathrm{P}<0.05)$.

The mRNA levels of M3 receptor in the B00 group was 0.6, 9.8 and 2.5-fold of that in the sham group at 3,6 and 12 weeks $(\mathrm{P}<0.05)$. At 3 weeks, there was no difference between the BOO with and without antimuscarinics group for M3 receptor expression $(\mathrm{P}>0.05)$. Solifenacin and Darifenacin could significantly suppress M3 receptor expressions at 6 and 12 
Fig. 10. The protein level of $\mathrm{M}$ receptor. Western blot analysis of the M2 and M3 receptor in bladder smooth muscle with GAPDH as the loading control. Data are given as mean \pm s.d, ${ }^{*} \mathrm{p}<0.05$ versus $B 00, \# p<0.05$ versus sham, $n=5$.

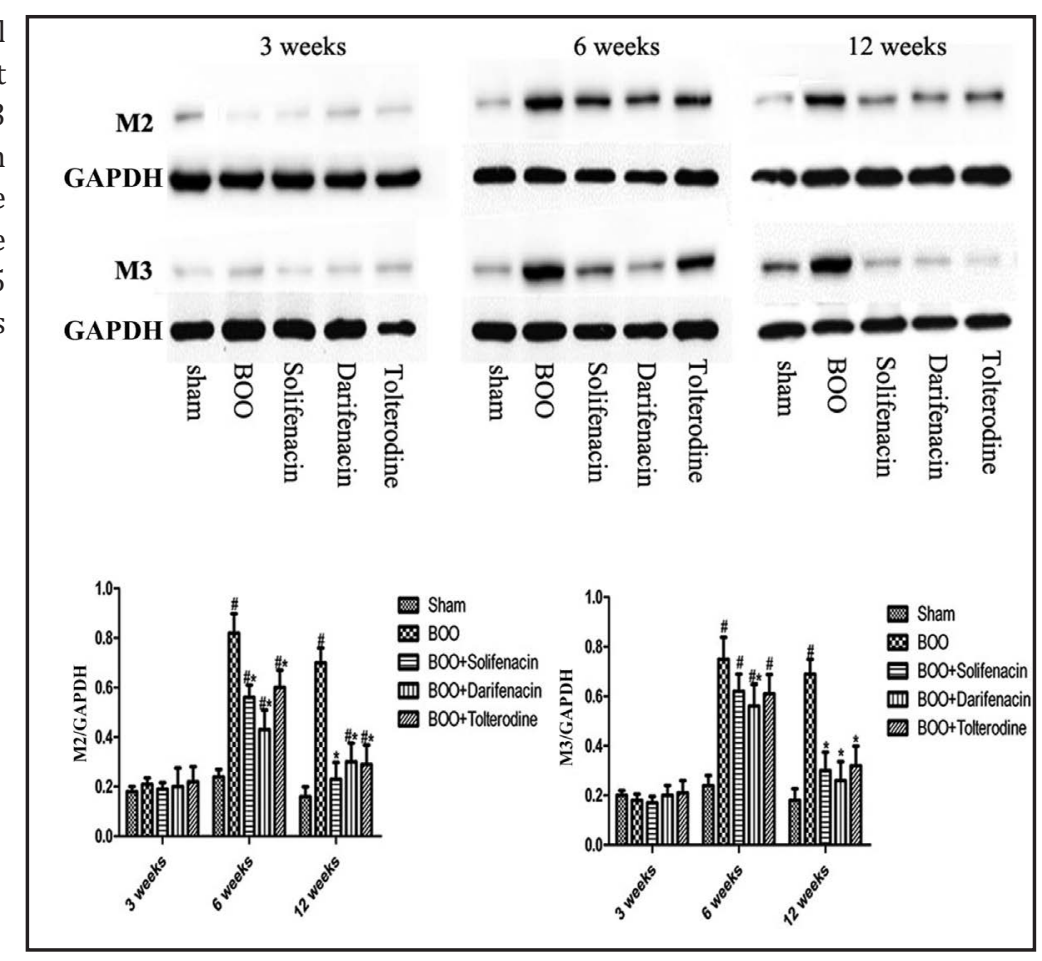

weeks $(\mathrm{P}<0.05)$. However, there was no significant difference in the $\mathrm{M} 3$ receptor expressions between the BOO with Tolterodine group and BOO group at 6 and 12 weeks $(\mathrm{P}>0.05)$. The mRNA level of M3 receptor was higher than that in the Darifenacin and Solifenacin group at 6 and 12 weeks. The result was also confirmed by Western blot experiment (Fig. 9, 10).

\section{Discussion}

Presently, there are many drugs that can relieve the bladder symptoms $[4,10]$. However, their effects on bladder structure and function have rarely been investigated. BOO is a major cause of progressive morphological and functional alterations within the urinary bladder and might cause bladder overactivity in some patients. Antimuscarinic drugs are effective for the relief of the symptoms of bladder overactivity. However, the effect of antimuscarinics on structural and functional changes was still unknown. Here, we found that M receptor antagonists lowered the proliferation of bladder cells under cyclic stretch in vitro. The research in vivo showed that the BOO with antimuscarinics group had lower weight of the bladder wall and less collagen deposition in muscle layer compared to the BOO group. The BOO with antimuscarinics group also had longer micturition interval in cytometric study and stronger contractility of muscle strip. The result also showed the antimuscarinics decrease the proliferation and inflammatory cytokines (CCL2, CCL5 and IL6) in smooth muscle cell. Therefore, this study demonstrated that antimuscarinics play a protective role in bladder remodeling within $\mathrm{BOO}$ situation.

Smooth muscle cells are very responsive to mechanical overload. Under abnormal or higher mechanical load, the smooth muscle cell will change its phenotypic type. The changed phenotypic type was able to proliferate [11]. In our study, the higher mechanical load induced the proliferation of bladder smooth muscle cell both in vitro and in vivo which could be inhibited by antimuscarinics. In previous study, Park also demonstrated that oxybutynin chloride could inhibit bladder smooth muscle cell proliferation induced by mechanical stretch [12]. However, in the BOO model, the proliferation occurred mainly at 3 weeks. Previous animal studies also reported that the DNA synthesis in the smooth muscle after obstruction 


\section{Cellular Physiology Cell Physiol Biochem 2017;44:907-919

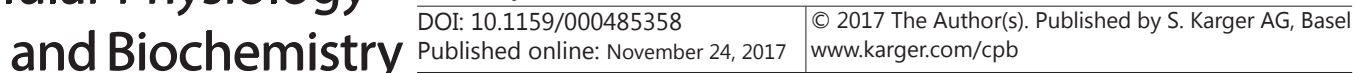 \\ Liu et al.: Antimuscarinics Prevent Bladder Remodeling}

occurred in the early phase of obstruction [13-15]. At 6 and 12 weeks, no significant difference was found in cell proliferation in 5 groups. Thus we think the proliferation of the bladder cells was an early biological change and early use of antimuscarinics may inhibit bladder hypertrophy caused by bladder cell proliferation. Another change of bladder cells in BOO was the ability to secrete extracellular matrix [11]. At 3 and 6 weeks, the percent collagen area was lower than that in the sham group. This was possibly because the proliferation and hypertrophy of bladder smooth muscle cells were more obvious at 3 and 6 weeks. At 12 weeks, the bladder was decompensated and significantly increased collagen deposition was found within the bladder wall. In our study, antimuscarinics could suppress collagen deposition as showed by Masson's trichrome. Further study was needed to examine the exact role of antimuscarinics on extracellular matrix changes in BOO.

Inflammatory responses were reported to be involved in $\mathrm{BOO}$ and partly responsible for bladder remodeling [16-19]_ENREF_22. In our study, we found that the expressions of CCL2, CCL5, and IL-6 gene were upregulated in BOO, and could be inhibited by antimuscarinics. Previous studies reported that inflammatory cytokines can cause sensitization of polymodal $C$-fibers and facilitate $A \beta$ input to the spinal cord leading to overactivity of the bladder [20, 21]. Arms reported that CCL2 expression was elevated in CYP-induced cystitis in rat and blockage of its high-affinity receptor was associated with increased bladder capacity and decreased void frequency [22]. In our study, antimuscarinics attenuated the CCL2, CCL5, and IL-6 expressions and the BOO with antimuscarinics groups had significantly longer micturition interval. So we think that the inflammatory component_ENREF_22 is important in the bladder remolding process and the attenuation of the inflammatory component ENREF_22 may at least partly contribute to the improvement of bladder function of the antimuscarinics treated rats.

Muscarinic receptor is an important contractile mediator in the bladder. In our study, the rats subjected to antimuscarinics were associated with improved bladder structure and function. Therefore, we think muscarinic receptor might also be an important mediator in the bladder remodeling after B00. The bladder expresses mainly the M2 and the M3 subtype of muscarinic receptors, and M2 subtype is expressed more prominently [23]. In present study, the change of M2 and M3 receptor expression after BOO was associated with duration of obstruction. They decreased at 3 weeks, and then increased at 6 and 12weeks.In the BOO with antimuscarinics group, the M receptors expressions were lower compared to the BOO group. We think the reversed pathophysiological changes by Antimuscarinics might be through direct antagonistic effect and reduced $M$ receptors expressions.

In our study, three muscarinic receptor antgonists were used. Darifenacin and solifenacin are selective for the M3 subtype. Tolterodine is nonselective for the M2 and M3 receptor. All the antimuscarinics could reverse the structural and functional changes of BOO bladder. However, we didn't notice any significant difference in the response to different antimuscarinics. As only one dosage for each drug was used and the maximal effect was not known, we still could not conclude whether M2 receptor played a role in the remodeling of B00. In the airway, allergen-induced changes were demonstrated to be via the muscarinic M3 receptor, and not via M1 or M2 receptors [24]. In bladder remodeling, however, further research is needed to determine the actual effect of each subtype.

\section{Conclusion}

Antimuscarinics could prevent the structural and functional changes of bladder wall, which can decrease the detrusor muscle hypertrophy and extracellular matrixc deposition with improvement of storage function and detrusor contractility. Patients with BOO will gain more benefit if they used antimuscarinics early. 


\section{Cellular Physiology Cell Physiol Biochem 2017;44:907-919 \begin{tabular}{l|l} 
and Biochemistry & DOI: 10.1159/000485358 \\
(c) 2017 The Author(s). Published by S. Karger AG, Basel \\
www.karger.com/cpb
\end{tabular} \\ Liu et al.: Antimuscarinics Prevent Bladder Remodeling}

\section{Acknowledgements}

This study was supported by Grant No. 81770673, No.31170907, No. 31370951, and No. 81470927 from the National Natural Science Foundation of China, Grant No. 2014SCU04B21 from Fund for Distinguished Young Scholars of Sichuan University, Grant No. JH2014053 from Academic Leader Training Fund of Sichuan Province and Grant No. JH2015017 from Application-oriented Foundation of Committee Organization Department of Sichuan Provincial Party.

\section{Disclosure Statement}

The authors declare no conflict of interest.

\section{References}

1 Abrams P, Andersson KE, Birder L, Brubaker L, Cardozo L, Chapple C, Cottenden A, Davila W, de Ridder D, Dmochowski R, Drake M, Dubeau C, Fry C, Hanno P, Smith JH, Herschorn S, Hosker G, Kelleher C, Koelbl H, Khoury S, Madoff R, Milsom I, Moore K, Newman D, Nitti V, Norton C, Nygaard I, Payne C, Smith A, Staskin D, Tekgul S, Thuroff J, Tubaro A, Vodusek D, Wein A, Wyndaele JJ: Fourth International Consultation on Incontinence Recommendations of the International Scientific Committee: Evaluation and treatment of urinary incontinence, pelvic organ prolapse, and fecal incontinence. Neurourol Urodyn 2010;29:213-240.

-2 Chapple CR, Roehrborn CG: A shifted paradigm for the further understanding, evaluation, and treatment of lower urinary tract symptoms in men: focus on the bladder. Eur Urol 2006;49:651-658.

-3 Platz EA, Joshu CE, Mondul AM, Peskoe SB, Willett WC, Giovannucci E: Incidence and progression of lower urinary tract symptoms in a large prospective cohort of United States men. J Urol 2012;188:496-501.

\$4 Novara G, Galfano A, Ficarra V, Artibani W: Anticholinergic drugs in patients with bladder outlet obstruction and lower urinary tract symptoms: A systematic review. Eur Urol 2006;50:675-683.

5 Yamaguchi 0: Latest treatment for lower urinary tract dysfunction: therapeutic agents and mechanism of action. Int J Urol 2013;20:28-39.

6 Gosens R, Zaagsma J, Meurs H, Halayko AJ: Muscarinic receptor signaling in the pathophysiology of asthma and COPD. Respir Res 2006;7:73.

7 Gosens R, Nelemans SA, Grootte Bromhaar MM, McKay S, Zaagsma J, Meurs H: Muscarinic M3-receptors mediate cholinergic synergism of mitogenesis in airway smooth muscle. Am J Respir Cell Mol Biol 2003;28:257-262.

-8 Krymskaya VP, Orsini MJ, Eszterhas AJ, Brodbeck KC, Benovic JL, Panettieri RA, Jr., Penn RB: Mechanisms of proliferation synergy by receptor tyrosine kinase and G protein-coupled receptor activation in human airway smooth muscle. Am J Respir Cell Mol Biol 2000;23:546-554.

-9 Suzaki I, Asano K, Shikama Y, Hamasaki T, Kanei A, Suzaki H: Suppression of IL-8 production from airway cells by tiotropium bromide in vitro. Int J Chron Obstruct Pulmon Dis 2011;6:439-448.

10 Pyo JS, Cho WJ: Systematic Review and Meta-Analysis of Intravesical Hyaluronic Acid and Hyaluronic Acid/ Chondroitin Sulfate Instillation for Interstitial Cystitis/Painful Bladder Syndrome. Cell Physiol Biochem 2016;39:1618-1625.

11 Yu G, Bo S, Xiyu J, Enqing X: Effect of bladder outlet obstruction on detrusor smooth muscle cell: an in vitro study. J Surg Res 2003;114:202-209.

12 Park JM, Bauer SB, Freeman MR, Peters CA: Oxybutynin chloride inhibits proliferation and suppresses gene expression in bladder smooth muscle cells. J Urol 1999;162:1110-1114.

13 Saito M, Longhurst PA, Murphy M, Monson FC, Wein AJ, Levin RM: 3H-thymidine uptake by the rat urinary bladder after partial outflow obstruction. Neurourol Urodyn 1994;13:63-69.

14 Monson FC, McKenna BA, Wein AJ, Levin RM: Effect of outlet obstruction on 3H-thymidine uptake: a biochemical and radioautographic study. J Urol 1992;148:158-162. 


\section{Cellular Physiology Cell Physiol Biochem 2017;44:907-919 \begin{tabular}{l|l} 
DOI: 10.1159/000485358 & $\begin{array}{l}\text { O 2017 The Author(s). Published by S. Karger AG, Basel } \\
\text { www.karger.com/cpb }\end{array}$ \\
\hline
\end{tabular}}

Liu et al.: Antimuscarinics Prevent Bladder Remodeling

15 Levin RM, Haugaard N, Levin SS, Buttyan R, Chen MW, Monson FC, Wein AJ: Bladder function in experimental outlet obstruction: pharmacologic responses to alterations in innervation, energetics, calcium mobilization, and genetics. Adv Exp Med Biol 1995;385:7-19.

16 Lin WY, Hsieh CC, Yang TY, Chen ML, Huang LY, Lin YP, Chang PJ, Levin RM, Wei YH: Transient increase in circulating myeloid-derived suppressor cells after partial bladder outlet obstruction. J Urol 2014;192:15691573.

17 Metcalfe PD, Wang J, Jiao H, Huang Y, Hori K, Moore RB, Tredget EE: Bladder outlet obstruction: progression from inflammation to fibrosis. BJU Int 2010;106:1686-1694.

18 Oka M, Fukui T, Ueda M, Tagaya M, Oyama T, Tanaka M: Suppression of bladder oxidative stress and inflammation by a phytotherapeutic agent in a rat model of partial bladder outlet obstruction. J Urol 2009;182:382-390.

19 Kanno Y, Mitsui T, Kitta T, Moriya K, Tsukiyama T, Hatakeyama S, Nonomura K: The inflammatory cytokine IL-1beta is involved in bladder remodeling after bladder outlet obstruction in mice. Neurourol Urodyn 2016;35:377-381.

20 Dray A: Inflammatory mediators of pain. Br J Anaesth 1995;75:125-131.

21 Baba H, Doubell TP, Woolf CJ: Peripheral inflammation facilitates Abeta fiber-mediated synaptic input to the substantia gelatinosa of the adult rat spinal cord. J Neurosci 1999;19:859-867.

22 Arms L, Girard BM, Malley SE, Vizzard MA: Expression and function of CCL2/CCR2 in rat micturition reflexes and somatic sensitivity with urinary bladder inflammation. Am J Physiol Renal Physiol 2013;305:F111-122.

23 Goepel M, Gronewald A, Krege S, Michel MC: Muscarinic receptor subtypes in porcine detrusor: comparison with humans and regulation by bladder augmentation. Urol Res 1998;26:149-154.

-24 Kistemaker LE, Bos ST, Mudde WM, Hylkema MN, Hiemstra PS, Wess J, Meurs H, Kerstjens HA, Gosens R: Muscarinic M(3) receptors contribute to allergen-induced airway remodeling in mice. Am J Respir Cell Mol Biol 2014;50:690-698. 